\title{
Polymorphisms CYP2R1 rs10766197 and CYP27B1 rs10877012 in Multiple Sclerosis: A Case-Control Study
}

\author{
A. Martinez-Hernandez $\mathbb{D},{ }^{1}$ E. E. Perez-Guerrero $\mathbb{D},^{2}$ M. A. Macias-Islas $\left(\mathbb{D},^{3}\right.$ \\ C. A. Nava-Valdivia $\mathbb{D}^{4},{ }^{4}$ A. Villagomez-Vega $\mathbb{D},{ }^{5}$ B. Contreras-Haro $\mathbb{D},{ }^{5}$ \\ Y. E. Garcia-Ortega $\mathbb{D}^{6}{ }^{6}$ Y. Esparza-Guerrero ${ }^{1},{ }^{1}$ S. G. Gallardo-Moya $\mathbb{D}^{1}{ }^{1}$ \\ J. I. Gamez-Nava $\mathbb{D}^{1,}{ }^{1,7}$ L. Gonzalez-Lopez $\mathbb{D},{ }^{1,7}$ E. Oliva-Flores, ${ }^{1}$ N. A. Rodriguez-Jimenez $\mathbb{D},^{8}$ \\ F. Cortes-Enriquez $\mathbb{D}^{9}{ }^{9}$ and A. M. Saldaña-Cruz $\mathbb{D}^{8}$
}

${ }^{1}$ Programa de Doctorado en Farmacología, Departamento de Fisiología, Centro Universitario de Ciencias de la Salud, Universidad de Guadalajara, Guadalajara, Mexico

${ }^{2}$ Instituto de Investigación en Ciencias Biomédicas, Centro Universitario de Ciencias de la Salud, Universidad de Guadalajara, Guadalajara, Jalisco, Mexico

${ }^{3}$ Departamento de Neurociencias, Centro Universitario de Ciencias de la Salud, Universidad de Guadalajara, Guadalajara, Jalisco, Mexico

${ }^{4}$ Departamento de Microbiología Y Patología, Centro Universitario de Ciencias de la Salud, Universidad de Guadalajara, Guadalajara, Jalisco, Mexico

${ }^{5}$ Departamento de Ciencias Biomédicas, Centro Universitario de Tonalá, Universidad de Guadalajara, Tonalá, Mexico

${ }^{6}$ Hospital de Especialidades, Centro Médico Nacional de Occidente, Guadalajara, Jalisco, Mexico

${ }^{7}$ Doctorado en Salud Pública, Departamento de Salud Pública, Centro Universitario de Ciencias de la Salud, Universidad de Guadalajara, Guadalajara, Jalisco, Mexico

${ }^{8}$ Instituto de Terapéutica Experimental Y Clínica, Departamento de Fisiología, Centro Universitario de Ciencias de la Salud, Universidad de Guadalajara, Guadalajara, Jalisco, Mexico

${ }^{9}$ Instituto Mexicano del Seguro Social, Guadalajara, Jalisco, Mexico

Correspondence should be addressed to A. M. Saldaña-Cruz; ana.saldanac@academicos.udg.mx

Received 29 October 2021; Accepted 17 November 2021; Published 23 December 2021

Academic Editor: Vlad Padureanu

Copyright (c) 2021 A. Martinez-Hernandez et al. This is an open access article distributed under the Creative Commons Attribution License, which permits unrestricted use, distribution, and reproduction in any medium, provided the original work is properly cited.

Background. Multiple sclerosis (MS) is a chronic autoimmune inflammatory disease. Low vitamin D levels have been reported to be a risk factor for MS, and genetic variances could be implicated. The aim of this study was to evaluate the association of MS with rs10766197 polymorphism of CYP2R1 gene and rs10877012 polymorphism of CYP27B1 gene. The second aim was to analyse whether these polymorphisms are associated with the severity of the progression of MS. Material and Methods. In a case-control study, we included 116 MS patients and 226 controls, all of whom were Mexican Mestizo. MS was diagnosed by McDonald criteria (2017). A complete neurological evaluation was performed to evaluate the severity of disease progression. Serum 25hydroxyvitamin D [25(OH) vitamin D] levels were measured by ELISA. Single nucleotide polymorphisms rs10766197 of CYP2R1 gene and rs10877012 SNP of CYP27B1 gene were genotyped by real-time PCR. Results. Serum 25(OH) vitamin D levels were lower in MS patients than in controls $(p=0.009)$. No differences were observed between serum $25(\mathrm{OH})$ vitamin $\mathrm{D}$ levels of MS patients with severe progression compared to low progression $(p=0.88)$. A higher frequency of the A allele of CYP2R1 rs10766197 was observed between MS patients and controls $(p=0.05)$. No differences were observed in the frequency of T allele of CYP27B1 rs10877012 ( $p=0.65)$. In subanalysis, patients with GA + AA genotypes of CYP2R1 rs10766197 had an increased risk of MS compared to controls $(p=0.03)$. No increased risk was observed in GT + TT genotypes of CYP27B1 rs10877012 
$(p=0.63)$. No differences were observed in allele frequencies of either polymorphism between patients with severe vs. low disease progression. Conclusion. Lower serum 25(OH) vitamin D levels were observed in MS patients than in controls, although these levels were not associated with disease progression. Carriers of GA + AA genotypes of CYP2R1 rs10766197 had an increased risk of MS. None of these polymorphisms was associated with severe progression of MS.

\section{Introduction}

Multiple sclerosis (MS) is a chronic inflammatory demyelinating disease of the central nervous system (CNS) [1]. Typically, MS affects the brain, spinal cord, and optic nerves, with inflammatory lesions derived from probable autoimmune mechanisms; these lesions are followed by axonal and grey-matter neurodegenerative processes associated with progressive worsening of disability. Most patients experience their first symptoms between the ages of 20 and 40 years. MS is considered the leading cause of disability in young people, excluding injuries caused by traffic accidents [2]. In Mexico, the prevalence of MS is 12 to 15 per 100,000 inhabitants [3].

There are four categories of MS: clinically isolated syndrome (CIS), relapsing-remitting (RRMS), secondary progressive (SPMS), and primary progressive (PPMS), with RRMS accounting for $85 \%$ of patients [4]. The World Health Organization (WHO) estimates that more than two million people worldwide suffer from MS [5].

Clinical symptoms of MS may include motor dysfunction, tremor, dysmetria, cerebellar ataxia, nystagmus, diplopia, hypoesthesia, blindness (usually unilateral), and sphincter disturbances such as retention or incontinence; approximately 45 to $60 \%$ of patients will develop cognitive decline and neuropsychiatric symptoms [6]. Motor disorders are the most common symptoms in the course of the disease which lead to motor disability [7].

MS is currently considered a very heterogeneous disorder in which both genetic susceptibility and environmental exposures are strongly implicated in $\mathrm{T}$ cell activation and pathogenesis [8]. Epstein-Barr virus, cytomegalovirus, and mononucleosis infections; smoking; adolescent obesity; night work; low sun exposure; and 25-hydroxyvitamin D $(25(\mathrm{OH})$ vitamin $\mathrm{D})<30 \mathrm{ng} / \mathrm{mL}$ are recognized as risk factors for MS [9]. Vitamin D has been proposed as a serum biomarker in several clinical conditions, including neurodegenerative disorders; additionally, several inflammatory and cardiovascular markers reflect the immunomodulatory function of vitamin D [10]. It has been hypothesized that lower 25(OH) vitamin D levels are associated with the risk of MS $[9,11]$. Possible explanations for the association between MS risk and altered vitamin $\mathrm{D}$ status alterations include the role of vitamin $\mathrm{D}$ in $\mathrm{CD} 4+\mathrm{T}$ cell balance and remyelination processes, B lymphocyte class switching, immunoglobulin production, and proinflammatory interleukin secretion $[12,13]$ as well as the presence of vitamin D-responsive elements (VDREs) in the promoter regions of various MS-associated genes [14].

The metabolism of vitamin D, or cholecalciferol, is carried out by enzymes of the cytochrome p 450 family, which catalyse a series of hydroxylation reactions to produce differ- ent active compounds [15]. CYP2R1 is responsible for the primary hepatic metabolism of vitamin $\mathrm{D}$ to $25(\mathrm{OH})$ vitamin $\mathrm{D}$, which is subsequently converted to 1,25 -dihydroxyvitamin $\mathrm{D}$, the primary bioactive vitamin $\mathrm{D}$ metabolite, by CYP27B1in the kidney [15-17]. By binding to the vitamin $\mathrm{D}$ receptor, a member of the nuclear receptor superfamily, this metabolite mediates functions in multiple classes of cells, including immune cells [18].

Therefore, it is of great importance to study genetic factors that may modify $25(\mathrm{OH})$ vitamin D levels and have consequential impacts on the disease. The CYP2R1 gene, found on chromosome 11p15.2, encodes the enzyme 25hydroxylase, which is involved in the first step of vitamin D hydroxylation activation. The rs 10766197 polymorphism of the CYP2R1 gene has been shown to be an important factor with a major impact on vitamin D levels $[19,20]$. The CYP27B1 gene is located on chromosome 12q13.1q13.3 and encodes the enzyme 1-alpha-hydroxylase. The rs10877012 polymorphism has been associated with reduced vitamin D levels; thus, together with the rs10766197 polymorphism, it is a promising object of research due to its potential influence in patients with MS (Ramos 2008) [20].

Prior research has found an association the rs 10766197 SNP of the CYP2R1 gene and the risk of MS as well as the progression of the disease [19]; but there has been no prior research on the rs10877012 SNP of the CYP27B1 gene in patients with MS.

Therefore, the first aim of this study was to evaluate the association of MS with the rs10766197 polymorphism of the CYP2R1 gene and the rs10877012 polymorphism of the CYP27B1 gene. The second aim was to analyse whether these polymorphisms are associated with the severity of MS progression.

\section{Materials and Methods}

2.1. Study Design. The design of this study is a Case-Control Study, in which the cases were patients with RRMS and the controls were healthy patients without MS, residents of the Guadalajara metropolitan area.

2.2. Clinical Setting. This study included 116 RRMS patients and 226 controls. The MS cases were patients treated at the Mexican Association for Multiple Sclerosis A.C. in Guadalajara, Mexico, from January 24, 2019, to August 31, 2021.

2.3. Study Subjects. All individuals were aged $\geq 18$ years and were Mexican-Mestizo as defined by the Mexican National Institute of Anthropology and History: "individuals who were born in Mexico, of the original autochthonous inhabitants of the region and individuals who were mainly Spaniards" [21]. 
Controls were selected randomly from the population available at the Clinical and Experimental Therapeutic Institute, consisting of 185 women and 41 men. Controls were included if they had no history of inflammatory or autoimmune disorders, and only one person per family was recruited. Controls were included to compare serum $25(\mathrm{OH})$ vitamin $\mathrm{D}$ levels and the genotypes of the polymorphisms.

2.4. Clinical Assessments. Patients with RRMS were included and assessed by an experienced neurologist and based on a previous history of disease and physical examination. Complete neurological examination assured that the patients fulfilled the 2017 McDonald clinical criteria. We excluded patients with kidney disease, liver disease, other uncontrolled autoimmune or psychiatric diseases, and current pregnancy.

Functional systems assessment was used to establish the disability score according to Kurtzke's Extended Disability Status Scale (EDSS) [22]. To ensure a correct diagnosis, we performed 1.5-tesla MRI with conventional T1, gadoliniumenhanced $\mathrm{T} 1, \mathrm{~T} 2$, and fluid-attenuated inversion recovery (FLAIR) sequences and confirmed the presence of typical round hyperintense lesions in T2 and FLAIR sequences and hypointensity in $\mathrm{T} 1$ with or without enhancement distributed in a different location; the time of evolution differed for each patient [23]. Oligoclonal bands were not considered mandatory to support or exclude an MS diagnosis [24]. Disability was measured using EDSS scores [22]. Disease progression was assessed using the progression index (PI). This index was calculated by dividing the degree of disability, as measured using the EDSS, by the duration of the disease; the typical PI value is 0.4-0.6 [25]. We used the upper end of this range as a cut-off point to dichotomize MS patients into two groups: $\mathrm{PI}>0.6$ (severe progression) and $\mathrm{PI} \leq 0.6$ (low progression).

Two experienced and trained researchers recorded the clinical and epidemiological characteristics of the participants, which included age, sex, treatment, duration of MS, and PI.

2.5. Genotyping CYP2R1 rs10766197 and CYP27B1 rs10877012 Polymorphisms. Peripheral whole blood samples, harvested in Vacutainer tubes containing ethylenediaminetetraacetic acid as an anticoagulant, were collected from each patient. Genomic DNA was obtained from these blood samples using the modified Miller technique [26]. Genomic DNA was quantified using a Nanodrop Genomic, and the DNA was diluted in Tris-EDTA buffer to $20 \mathrm{ng} / \mu \mathrm{L}$ and placed in $200 \mu \mathrm{L}$ propylene cryotubes (Eppendorf ${ }^{\mathrm{TM}}$ ). Genotyping of CYP2R1 rs10766197 and CYP27B1 rs10877012 polymorphisms was performed by quantitative polymerase chain reaction (qPCR) using TaqMan probes [27]. TaqMan Assay IDs C_2958435_10 and C_26237740_ 10 were performed according to the manufacturer's instructions (Applied Biosystems); the StepOne ${ }^{\mathrm{TM}}$, Real-Time polymerase chain reaction (qPCR) system was employed for this purpose (Applied Biosystems). All results were independently scored by two investigators blinded to patient information. In case of ambiguous results, the sample was analysed a second time.
2.6. Quantification of Serum 25(OH) Vitamin D Levels. A peripheral venous blood sample was taken from each patient at the time of inclusion. Serum levels of $25(\mathrm{OH})$ vitamin D were quantified by ELISA using commercial kits (My BioSource; MBS580159, San Diego, CA, USA). All procedures were performed according to the manufacturer's recommendations. All measurements were performed by the same researchers, who were blinded to the clinical characteristics of the patients to avoid measurement biases.

2.7. Statistical Analysis. Qualitative variables were expressed as frequencies and percentages. Quantitative variables are expressed as the median, minimum ( $\mathrm{min}$ ), and maximum (max).

We identified genotypic frequencies by direct counting. Allelic frequencies were determined by direct counting of the observed genotypic frequencies. The chi-square test (or Fisher's exact test if required) was used for the comparison of proportions. For variables that did not satisfy the assumptions of parametric tests, Mann-Whitney $U$ nonparametric tests were performed.

Odds ratio (OR) and the $95 \%$ confidence interval (95\% CI) were calculated for genetic characteristics. The Kruskal-Wallis test was performed to compare serum $25(\mathrm{OH})$ vitamin $\mathrm{D}$ levels between genotypes. We also performed post hoc tests with the Bonferroni correction for multiple comparisons.

Hardy-Weinberg equilibrium (HWE) for CYP2R1 rs10766197 and CYP27B1 rs10877012 polymorphisms was evaluated in the groups and was determined by comparing the observed and expected data using the chi-squared test.

We performed a multivariable logistic regression analysis to identify variables associated with the MS. This model was adjusted by confunders (age and gender) and genotypes for genes. For the CYP2R1 gene rs10766197 GG was stated as referent and $\mathrm{GA}+\mathrm{AA}$ was considered as risk. For the CYP27B1 gene rs10877012 GG was used as referent and GT + TT was selected as risk. The variables included in the model were selected using a stepwise method.

A $p$ value was considered significant at the $p \leq 0.05$ level. Data were analysed with R 4.1.0 base [28] and with the epiR [29] and ggplot2 packages [30].

2.8. Ethics and Consent. This study was approved by the Ethics Committee and Biosafety Committee from Centro Universitario de Ciencias de la Salud (CUCS) Universidad de Guadalajara, Jalisco, Mexico, with registration number CI-03519. This study was conducted according to the principles expressed in the Declaration of Helsinki. All study participants voluntarily provided written informed consent.

\section{Results}

We assessed 116 patients with MS and 226 individuals in the control group. The genotype distributions of the SNPs rs10766197 and rs10877012 in the control group were consistent with HWE (rs10766197 $p>0.05$ and rs10877012 $p>0.05$ ) (data not shown in tables).

The baseline characteristics of the MS patients are shown in Table 1. Of all MS patients included, $65.5 \%$ were females. 
TABLE 1: Sociodemographic and clinical descriptive characteristics in multiple sclerosis patients.

\begin{tabular}{lc}
\hline Variable & $\begin{array}{c}\text { Multiple sclerosis } \\
n=116\end{array}$ \\
\hline Female, $n$ (\%) & $76(65.5)$ \\
Age, (years) & $38(19-66)$ \\
Disease characteristics & \\
Disease evolution, (years) & $7.5(1.0-28.0)$ \\
EDSS, (score) & $3.0(0.0-7.0)$ \\
Progression index, (score) & $0.38(0.0-4.5)$ \\
$\quad$ Severe progression (IP > 0.6), $n(\%)$ & $39(33.6)$ \\
$\quad$ Low progression (IP $\leq 0.6), n(\%)$ & $77(66.4)$ \\
Serum 25(OH) vitamin D levels, (ng/mL) & $18.3(3.8-50.9)$ \\
Sufficient $(>30 \mathrm{ng} / \mathrm{mL}), n(\%)$ & $14(12.0)$ \\
Insufficient $(20-30 \mathrm{ng} / \mathrm{mL}), n(\%)$ & $17(14.7)$ \\
Deficient $(<20 \mathrm{ng} / \mathrm{mL}), n(\%)$ & $85(73.3)$ \\
Treatment & \\
Glatiramer acetate, $n(\%)$ & $39(33.6)$ \\
Interferon, $n(\%)$ & $27(23.3)$ \\
Rituximab, $n(\%)$ & $12(10.3)$ \\
Fingolimod, $n(\%)$ & $11(9.5)$ \\
Dimethyl fumarate, $n(\%)$ & $7(6.0)$ \\
Azathioprine, $n(\%)$ & $5(4.3)$ \\
Natalizumab, $n(\%)$ & $4(3.5)$ \\
No treatment, $n(\%)$ & $11(9.5)$ \\
\hline
\end{tabular}

EDSS: Expanded Disability Status Scale of Kurtzke for Multiple Sclerosis; IP: index progression (EDSS/disease evolution). Qualitative variables were expressed as frequency and percentage. Quantitative variables were expressed as median and min-max.

We observed a median age of 38 (19-66) years and a median of 7.5 (1-28) years of disease evolution. Overall, $33.6 \%$ of the patients with MS presented severe disease progression (PI $>0.6$ ), while $66.4 \%$ presented low progression of the disease (IP < 0.6). Deficiency of serum $25(\mathrm{OH})$ vitamin D levels in $73.3 \%$ of MS patients was observed. The most prescribed treatment in MS was glatiramer acetate (33.6\%), followed by interferons (23.3\%) and rituximab (10.3\%).

Table 2 presents the comparison of sociodemographic, serological, and genetic characteristics between MS patients and the control group. For rs10766197 of the CYP2R1 gene, we observed a higher frequency of the heterozygous genotype GA (50.0\%) in MS patients, while in the control group, we observed an increased frequency of the wild-type homozygous genotype GG (49.6\%); however, these differences were not statistically significant $(p=0.08)$. The A allele was observed at a higher frequency in MS patients than in the control group (37.9\% vs. $30.5 \%, p=0.05)$. For rs 10877012 of the CYP2B1 gene, we observed a similar frequency of genotypes in both groups. We did not identify a significant difference between MS patients and the control group $(p=0.90)$. Additionally, in a subgroup of 106 individuals in the control group, we compared the $25(\mathrm{OH})$ vitamin $\mathrm{D}$ levels. Higher levels of $25(\mathrm{OH})$ vitamin $\mathrm{D}$ were observed in the control group than in the MS group $(p=0.009)$ (Figure 1). We identified a higher percentage of individuals who had sufficient $25(\mathrm{OH})$ vitamin D levels in the control group $(43.4 \%, p=0.003)$ and a higher percentage of individuals deficient in vitamin D in MS patients $(73.3 \%, p<0.001)$.

Table 3 shows the comparison of clinical characteristics between severe progression versus low progression in MS patients. We observed the same proportion of females in the severe progression and low progression groups $(64.1 \%$ vs. $65.1 \%$, respectively). We observed a shorter disease duration in the severe progression group than in the low progression group ( 3 years vs. 12 years, $p<0.001$ ). In quantitative serum $25(\mathrm{OH})$ vitamin D levels, we did not observe a significant difference between the severe and low progression groups. In a stratified comparison of serum $25(\mathrm{OH})$ vitamin D levels, we observed a higher percentage of individuals with deficient levels $(<20 \mathrm{ng} / \mathrm{mL})$ and found a significant difference between groups $(p=0.037)$. No differences were observed in genotypes and allele frequencies of either polymorphism between patients with severe vs. low disease progression.

Table 4 compares the genotypic and allelic frequencies of polymorphisms rs10766197 and rs10877012 between MS patients and the control group. In a comparison of the GG, GA, and AA genotypes of the rs10766197 polymorphism of CYP2R1 between MS patients and the control group, a trend to almost significant differences were observed $(p=0.08)$. In a comparison of the GG, GT, and TT genotypes of the rs10877012 polymorphism of CYP2B1 between MS patients and the control group, no differences were observed. OR and their 95\% CI were calculated for the comparison between dominant and recessive genetic models. We observed a high risk of MS in the dominant model of rs10766197 of the CYP2R1 gene $(\mathrm{OR}=1.67 ; 95 \% \mathrm{CI}=1.05-2.64 ; p=0.02)$. No differences in the risk of other genetic models or in rs 10877012 of the CYP2B1 gene polymorphism were observed.

Distribution analysis of serum $25(\mathrm{OH})$ vitamin D levels according to the genotypes of polymorphisms stratified between MS patients and the control group revealed that patients who carried the genotype GG for rs10766197 presented higher levels, with a median of $17.44 \mathrm{ng} / \mathrm{mL}$ (ranges of $8.45-45.32 \mathrm{ng} / \mathrm{mL}$ ).

In data not shown, we performed a multivariable logistic regression analysis. After adjusting by confounders variables, we found that age $(\mathrm{OR}=0.95$, CI 95\% 0.92-0.98; $p=0.001)$ and gender ratio $(\mathrm{OR}=2.18, \mathrm{CI} 95 \% 1.16-4.2 ; p=0.017)$ were associated with an increase of risk for MS. The GA + AA genotypes of CYP2R1 rs10766197 polymorphism were associated with an increase of $\mathrm{MS}(\mathrm{OR}=1.87$, CI $95 \%$ 1.07-3.29; $p=0.02)$. However, the CYP27B1 rs10877012 polymorphism was not associated with the disease.

In the comparison of serum $25(\mathrm{OH})$ vitamin $\mathrm{D}$ levels between GG, GA, and AA genotypes, we observed significant differences $(p=0.046)$, and in the post hoc analysis, a notably significant difference was observed between the GA and AA genotypes $(p=0.029)$. In the comparison of serum $25(\mathrm{OH})$ vitamin D levels between genotypes of rs10877012 of the CYP2B1 gene, we did not observe a significant difference $(p=0.94)$ (Figure 2) (data not shown in tables).

In the comparison of genotypic and allelic frequencies of polymorphisms rs10766197 of the CYP2R1 gene and rs10877012 of the CYP27B1 gene between severe 
TABLE 2: Comparison of sociodemographic, serological, and genetic characteristics of multiple sclerosis patients versus control group.

\begin{tabular}{|c|c|c|c|}
\hline Variable & $\begin{array}{c}\text { Multiple sclerosis } \\
n=116\end{array}$ & $\begin{array}{c}\text { Control group } \\
n=226\end{array}$ & $p$ \\
\hline Female, $n(\%)$ & $76(65.5)$ & $185(81.9)$ & 0.001 \\
\hline Age (years) & $38(19-66)$ & $46.0(21-66)$ & $<0.001$ \\
\hline \multicolumn{4}{|l|}{ Serum levels } \\
\hline $25(\mathrm{OH})$ vitamin $\mathrm{D}$ levels $(\mathrm{ng} / \mathrm{mL}), n(\%)$ & $16.2(3.8-50.9)$ & $26.8(4.3-53.2) *$ & 0.009 \\
\hline Sufficient (>30 ng/mL), $n(\%)$ & $14(12.0)$ & $46(43.4) *$ & \\
\hline Insufficient (20-30 ng/mL), $n(\%)$ & $17(14.7)$ & $14(13.2) *$ & 0.009 \\
\hline Deficient $(<20 \mathrm{ng} / \mathrm{mL}), n(\%)$ & $85(73.3)$ & $46(43.4) *$ & \\
\hline \multicolumn{4}{|l|}{ Genetic characteristics } \\
\hline \multicolumn{4}{|l|}{ rs10766197, CYP2R1 gene } \\
\hline \multicolumn{4}{|l|}{ Genotypes } \\
\hline GG, $n(\%)$ & $43(37.1)$ & $112(49.6)$ & \\
\hline GA, $n(\%)$ & $58(50.0)$ & $90(39.8)$ & 0.08 \\
\hline $\mathrm{AA}, n(\%)$ & $15(12.9)$ & $24(10.6)$ & \\
\hline Allele $2 n=684$ & $2 n=232$ & $2 n=452$ & \\
\hline $\mathrm{A}, n(\%)$ & $88(37.9)$ & $138(30.5)$ & 0.05 \\
\hline $\mathrm{G}, n(\%)$ & $144(62.1)$ & $314(69.5)$ & 0.05 \\
\hline \multicolumn{4}{|l|}{ rs10877012, CYP2B1 gene } \\
\hline \multicolumn{4}{|l|}{ Genotypes } \\
\hline GG, $n(\%)$ & $55(47.4)$ & $101(44.7)$ & \\
\hline GT, $n(\%)$ & $48(41.4)$ & $98(43.4)$ & 0.90 \\
\hline TT, $n(\%)$ & $13(11.2)$ & $27(11.9)$ & \\
\hline Allele $2 n=684$ & $2 n=232$ & $2 n=452$ & \\
\hline $\mathrm{G}, n(\%)$ & $158(68.1)$ & $300(66.4)$ & 0.65 \\
\hline $\mathrm{T}, n(\%)$ & $74(31.9)$ & $152(33.6)$ & 0.65 \\
\hline
\end{tabular}

For rs10766197 of CYP2R1 gene: GG: wild homozygous; GA: heterozygous; AA: polymorphic homozygous. For rs10877012 of CYP2B1 gene: GG: wild homozygous; GT: heterozygous; TT: polymorphic homozygous. Qualitative variables were expressed as frequency and percentage. Quantitative variables were expressed as median and min-max. Comparisons between medians were performed using Mann-Whitney $U$ test. Comparison between proportions was performed using Chi-square test (or Fisher exact test if applicable). $p$ values were obtained comparing multiple sclerosis versus control group. $*$ Serum levels of 25(OH) vitamin D were determined in 107 healthy controls.

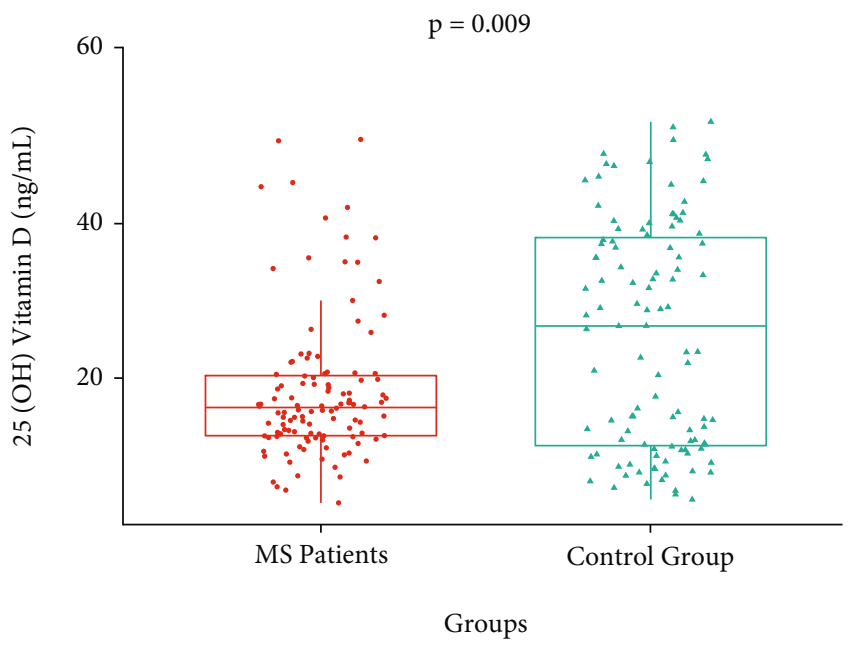

Figure 1: Levels of $25(\mathrm{OH})$ vitamin $\mathrm{D}$ in serum between MS patients and the control group $(p=0.009)$. The comparison was performed by Mann-Whitney $U$ test. progression vs. low progression in MS patients, we observed a similar frequency of the AA genotype for rs10766197 of the CYP2R1 gene (15.4\% in severe progression vs. $11.6 \%$ in low progression). Furthermore, for rs10877012 of CYP2B1 gene, we observed a higher frequency in of the heterozygous GT genotype in the severe group (48.4\%) than low progression group, in which we observed the wild homozygous GG with high frequency (51.9\%). No significant differences were observed in the comparison of genotypes between the severe and low MS progression groups (data not shown in tables).

\section{Discussion}

In this study, we observed that the rs10766197 polymorphism of the CYP2R1 gene confers a risk of MS. However, the rs10877012 polymorphism of the CYP27B1 gene was not associated with the risk of MS. We report the first allelic and genotypic frequencies in a Latin American country for the rs10766197 polymorphism of the CYP2R1 gene and the rs10877012 polymorphism of the CYP27B1 gene. 
TABLE 3: Comparison of clinical and genetic characteristics between severe progression and low progression in multiple sclerosis patients.

\begin{tabular}{|c|c|c|c|}
\hline Variable & $\begin{array}{l}\text { Severe progression } \\
\begin{array}{c}(\mathrm{IP}>0.6) \\
n=39\end{array}\end{array}$ & $\begin{array}{c}\text { Low progression } \\
\begin{array}{c}(\mathrm{IP} \leq 0.6) \\
n=77\end{array}\end{array}$ & $p$ \\
\hline Female, $n(\%)$ & $25(64.1)$ & $51(66.2)$ & 0.98 \\
\hline Age, (years) & $36(19-59)$ & $40(19-66)$ & 0.13 \\
\hline \multicolumn{4}{|l|}{ Disease characteristics } \\
\hline Disease evolution, (years) & $3(1-9)$ & $12(2-28)$ & $<0.001$ \\
\hline EDSS, (score) & $4.0(1-7)$ & $2.5(0-7)$ & 0.008 \\
\hline Serum $25(\mathrm{OH})$ vitamin D levels, $(\mathrm{ng} / \mathrm{mL})$ & $15.7(3.8-50.9)$ & $16.4(5.4-45.3)$ & 0.88 \\
\hline Sufficient (>30 ng/mL), $n(\%)$ & $4(10.3)$ & $13(16.9)$ & \\
\hline Insufficient $(20-30 \mathrm{ng} / \mathrm{mL}), n(\%)$ & $12(30.7)$ & $9(11.7)$ & 0.037 \\
\hline Deficient $(<20 \mathrm{ng} / \mathrm{mL}), n(\%)$ & $23(59.0)$ & $55(71.4)$ & \\
\hline \multicolumn{4}{|l|}{ Treatment } \\
\hline Glatiramer acetate, $n(\%)$ & $9(23.1)$ & $30(39.0)$ & 0.10 \\
\hline Interferon, $n(\%)$ & $7(17.9)$ & $20(25.9)$ & 0.36 \\
\hline Rituximab, $n(\%)$ & $9(23.1)$ & $3(3.9)$ & 0.003 \\
\hline Fingolimod, $n(\%)$ & $3(7.7)$ & $8(10.4)$ & 0.75 \\
\hline Dimethyl fumarate, $n(\%)$ & $4(10.3)$ & $3(3.9)$ & 0.22 \\
\hline Azathioprine, $n(\%)$ & $3(7.7)$ & $2(2.6)$ & 0.33 \\
\hline Natalizumab, $n(\%)$ & $1(2.5)$ & $3(3.9)$ & 1.00 \\
\hline No treatment, $n(\%)$ & $3(7.7)$ & $8(10.4)$ & 0.49 \\
\hline \multicolumn{4}{|l|}{ Genetic characteristics } \\
\hline \multicolumn{4}{|l|}{ rs10766197, CYP2R1 gene } \\
\hline \multicolumn{4}{|l|}{ Genotypes } \\
\hline GG, $n(\%)$ & $12(30.8)$ & $31(40.3)$ & \\
\hline $\mathrm{GA}, n(\%)$ & $21(53.8)$ & $37(48.0)$ & 0.58 \\
\hline $\mathrm{AA}, n(\%)$ & $6(15.4)$ & $9(11.7)$ & \\
\hline Allele $2 n=232$ & $2 n=78$ & $2 n=154$ & \\
\hline $\mathrm{A}, n(\%)$ & $33(42.3)$ & $55(35.7)$ & 0.33 \\
\hline $\mathrm{G}, n(\%)$ & $45(57.7)$ & $99(64.3)$ & 0.33 \\
\hline \multicolumn{4}{|l|}{ rs10877012, CYP2B1 gene } \\
\hline \multicolumn{4}{|l|}{ Genotypes } \\
\hline GG, $n(\%)$ & $15(38.5)$ & $40(51.9)$ & \\
\hline GT, $n(\%)$ & $19(48.7)$ & $29(37.7)$ & 0.39 \\
\hline $\mathrm{TT}, n(\%)$ & $5(12.8)$ & $8(10.4)$ & \\
\hline Allele $2 n=232$ & $2 n=78$ & $2 n=154$ & \\
\hline $\mathrm{T}, n(\%)$ & $49(62.8)$ & $45(29.2)$ & $<0.001$ \\
\hline $\mathrm{G}, n(\%)$ & $29(37.2)$ & $109(70.8)$ & $<0.001$ \\
\hline
\end{tabular}

EDSS: Expanded Disability Status Scale of Kurtzke for Multiple Sclerosis; IP: index progression (EDSS/disease evolution). For rs10766197 of CYP2R1 gene: GG: wild homozygous; GA: heterozygous; AA: polymorphic homozygous. For rs10877012 of CYP2B1 gene: GG: wild homozygous; GT: heterozygous; TT: polymorphic homozygous. Qualitative variables were expressed as frequency and percentage. Quantitative variables were expressed as median and minmax. Comparisons between medians were performed using Mann-Whitney $U$ test. Comparison between proportions was performed using Chi-square test (or Fisher exact test if applicable). $p$ values were obtained comparing multiple sclerosis with severe progression versus multiple sclerosis with low progression.

CYP2R1 is responsible for the hydroxylation of vitamin $\mathrm{D}$ to $25(\mathrm{OH})$ vitamin $\mathrm{D}$ in the first activation step. It is thought to be an important determinant of the vitamin $\mathrm{D}$ metabolic pathway, as it shows a high affinity for vitamin $D$ [31]. The SNP rs10766197 is located in the promoter region of the CYP2R1 gene, which causes differences in gene expression [32]. The association between the rs10766197 polymorphism of the CYP2R1 gene and MS has been previ- ously identified. Scazzone et al. (2017) reported that an association between the GA genotype (heterozygous minor allele carriers) in Italian patients was $46 \%$ in MS patients vs. $43 \%$ in controls (OR 2.19, 95\% CI 1.09-4.39, $p=0.03$ ), and the frequency of the AA genotype (homozygous minor allele carriers) was $39 \%$ in MS patients vs. $25 \%$ in controls (OR 3.18, 95\% CI 1.52-6.65, p 5 0.002) [19]. Other studies analysed other polymorphisms in this gene. Laursen et al. found 
TABLE 4: Comparison of genotypic and allelic frequencies of polymorphisms rs10766197 of CYP2R1 gene and rs10877012 of CYP2B1 gene, between multiple sclerosis patients and control group.

\begin{tabular}{|c|c|c|c|c|c|}
\hline & $\begin{array}{c}\text { Multiple sclerosis } \\
n=116\end{array}$ & $\begin{array}{c}\text { Control group } \\
n=226\end{array}$ & OR & $95 \% \mathrm{CI}$ & $p$ \\
\hline \multicolumn{6}{|l|}{ rs10766197, CYP2R1 gene } \\
\hline \multicolumn{6}{|l|}{ Genotypes } \\
\hline GG $n=155(\%)$ & $43(37.1)$ & $112(49.6)$ & - & - & \\
\hline GA $n=140(\%)$ & $58(50.0)$ & $90(39.8)$ & - & - & 0.08 \\
\hline AA $n=39(\%)$ & $15(12.9)$ & $24(10.6)$ & - & - & \\
\hline $\mathrm{GA}+\mathrm{AA}$ versus $\mathrm{GG}$ (as referent) & $73(62.9)$ & $114(50.4)$ & 1.67 & $1.05-2.64$ & 0.03 \\
\hline GA + GG versus AA (as referent) & $101(87.0)$ & $202(89.4)$ & 0.8 & $0.40-1.59$ & 0.59 \\
\hline Alleles $2 n=684$ & $2 n=232$ & $2 n=452$ & & & \\
\hline A allele, $2 \mathrm{n}=226(\%)$ & $88(37.9)$ & $138(30.5)$ & 1.39 & $0.99-1.94$ & 0.05 \\
\hline G allele, $2 n=458(\%)$ & $144(62.1)$ & $314(69.5)$ & 0.72 & $0.52-1.00$ & 0.05 \\
\hline \multicolumn{6}{|l|}{ rs10877012, CYP2B1 gene } \\
\hline \multicolumn{6}{|l|}{ Genotypes } \\
\hline GG $n=156(\%)$ & $55(47.4)$ & $101(44.7)$ & - & - & \\
\hline GT $n=146(\%)$ & $48(41.4)$ & $98(43.4)$ & - & - & 0.90 \\
\hline TT $n=40(\%)$ & $13(11.2)$ & $27(11.9)$ & - & - & \\
\hline GT + TT versus GG (as referent) & $61(52.6)$ & $125(55.3)$ & 0.89 & $0.57-1.40$ & 0.63 \\
\hline GG + GT versus TT (as referent) & $103(88.8)$ & $199(88.1)$ & 1.08 & $0.53-2.17$ & 0.84 \\
\hline Allele $2 n=684$ & $2 n=232$ & $2 n=452$ & & & \\
\hline G allele, $2 n=458(\%)$ & $158(68.1)$ & $300(66.4)$ & 1.08 & $0.77-1.52$ & 0.65 \\
\hline T allele, $2 n=226(\%)$ & $74(31.9)$ & $152(33.6)$ & 0.92 & $0.66-1.30$ & 0.65 \\
\hline
\end{tabular}

For rs10766197 of CYP2R1 gene: GG: wild homozygous; GA: heterozygous; AA: polymorphic homozygous; for rs10877012 of CYP2B1 gene: GG: wild homozygous; GT: heterozygous; TT: polymorphic homozygous. OR: odds ratio risk; $95 \%$ CI: $95 \%$ confidence interval. $p$ values were obtained comparing multiple sclerosis versus control group.

a significant association of CYP2R1 rs10741657 with MS risk in a cross-sectional study performed on the Danish population [33].

In our study, we found that the frequency of vitamin D deficiency was lower in MS patients than in controls. Our findings are supported by other studies; Oliveira et al., 2017 reported an association between serum levels of $25(\mathrm{OH})$ vitamin $\mathrm{D}$ and MS, reporting and media of MS patients $(26.12 \pm 8.47 \mathrm{ng} / \mathrm{mL})$ vs. controls $(29.71 \pm 9.17 \mathrm{ng} /$ $\mathrm{mL}, p=0.02$ ) [34], and Munger et al., 2006 found that the risk of MS decreased with increasing serum levels of $25(\mathrm{OH})$ vitamin D [35]. Several hypotheses have been proposed in advance to explain the role of vitamin $D$ in the MS. First, vitamin D influences the balance of CD4 T lymphocytes, by decreasing Th1 and Th17 cell differentiation and promoting Th2 and regulatory $\mathrm{T}$ cells (Tregs) proliferation. In the development of MS, CD4 T lymphocytes (or T helper lymphocytes, including Th1, Th2, Th17 subsets) play a crucial role in the activation of myelin-specific Th1 and Th17 cells, which drives the inflammation within the central nervous system $[36,37]$. Second, MS is characterized by progressive demyelination, vitamin $\mathrm{D}$ is deemed to have a role in the myelination and remyelination processes [38-41], and finally, $80 \%$ of MS-associated genes are enriched for VDREs in their promoter region and consequently, and they are regulated by vitamin $\mathrm{D}$ [42].
CYP27B1, encoding 1a-hydroxylase, which converts $25(\mathrm{OH})$ vitamin $\mathrm{D}$ to its active form, $1,25(\mathrm{OH}) 2 \mathrm{D}$, is the cytochrome P450 gene most strongly associated with vitamin D status [15]. The SNP rs10877012 located on the 5' untranslated region (UTR) may affect transcript stabilization and the posttranscriptional control of mRNA [43]. For the rs 10877012 polymorphism of the CYP27B1 gene, we observed that genotype or allele does not confer a clinically relevant risk for the development of MS. These results are in agreement with those reported by Simon et al. in American women with MS; in the study, findings do not support a role for an independent effect of the vitamin D-related gene polymorphisms investigated and the risk of MS [44]. The authors also identified three additional SNPs on the CYP27B1 gene associated with MS [19].

Genome-wide association studies (GWAS) and other epidemiological studies have shown that SNPs within the CYP2R1 gene modulate 25(OH) vitamin D levels [45-47]. In our study, we found that MS patients carrying one or two A alleles had lower $25(\mathrm{OH})$ vitamin D serum levels in comparison to patients carrying allele G. Scazzone et al. reported the rs10766197 distribution in MS patients, stratified according to the $25(\mathrm{OH})$ vitamin $\mathrm{D}$ concentrations, and the patients who carried the AA genotype presented a trend of lower levels of $25(\mathrm{OH})$ vitamin $\mathrm{D}$ in comparison to those with a genotype of GG or GA, although the difference was not statistically significant [14]. In contrast, other 


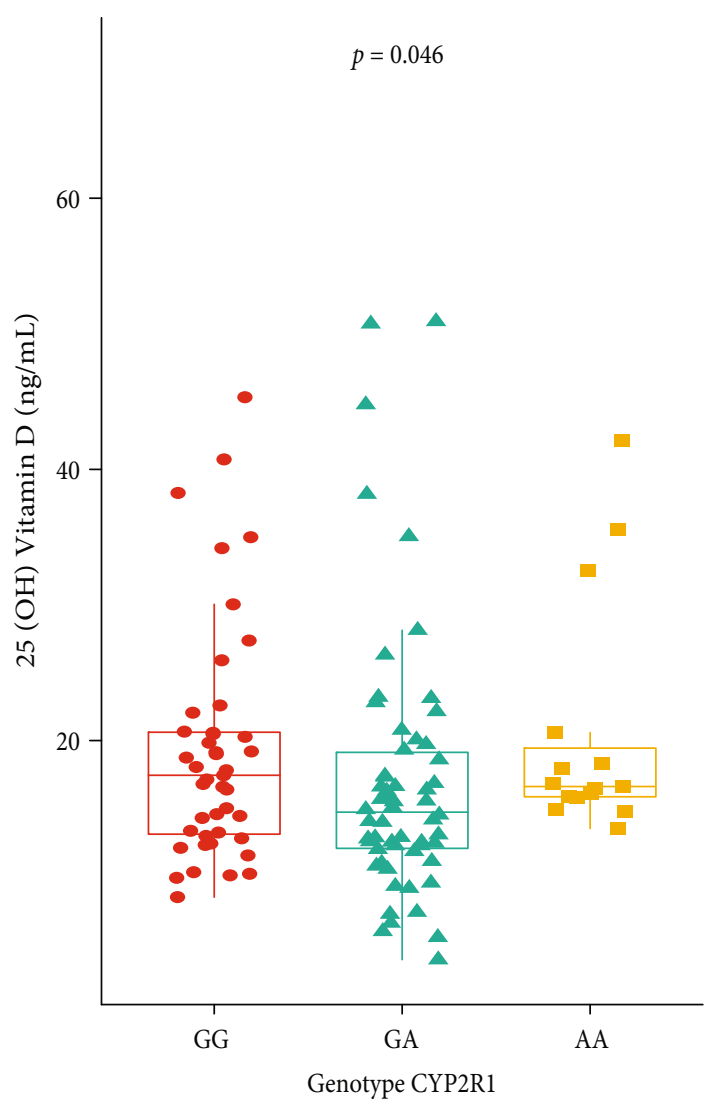

(a)

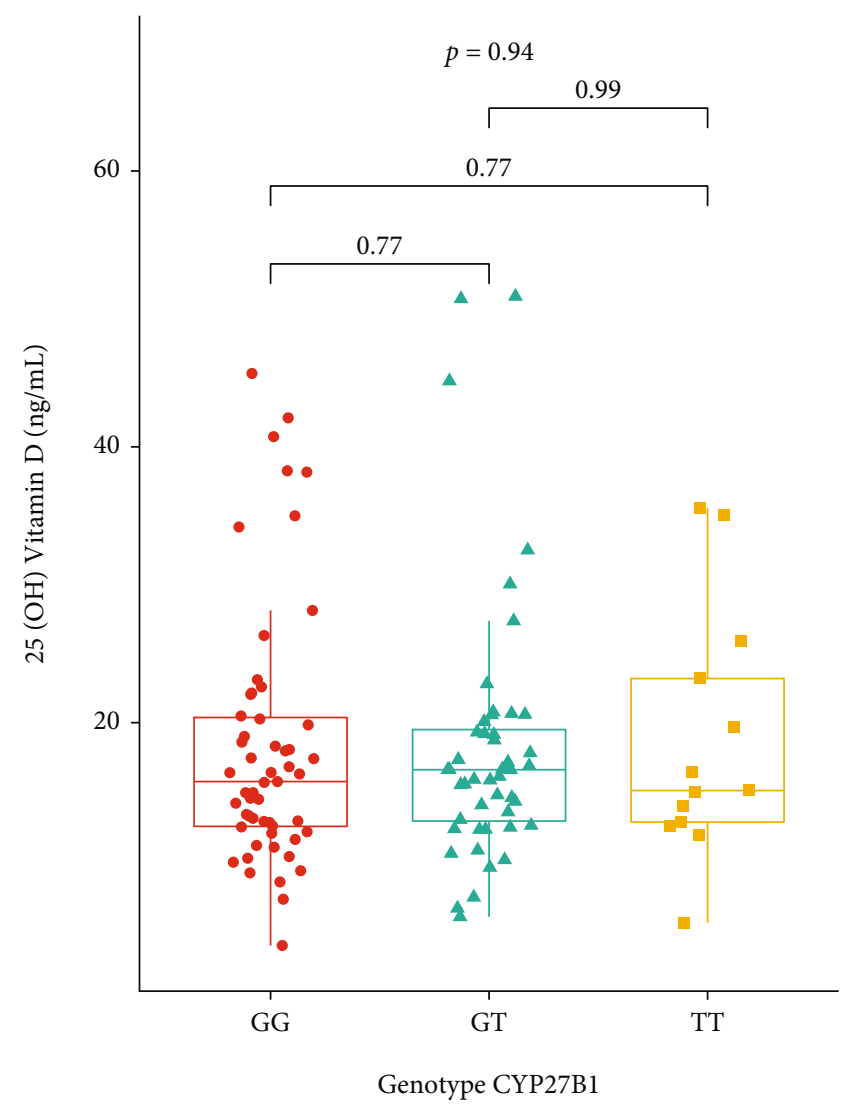

(b)

Figure 2: Comparison of levels of $25(\mathrm{OH})$ vitamin D in serum according to genotypes of single nucleotide polymorphisms rs10766197 of CYP2R1 gene and rs10877012 of CYP27B1 gene between MS patients. (a) Comparison of the levels of 25(OH) vitamin D in serum between genotypes of rs 10766197 of the CYP2R1 gene. (b) Comparison of the levels of 25(OH) vitamin D in serum between genotypes of rs10877012 of the CYP27B1 gene. Data are presented as medians with ranges (min-max) of $25(\mathrm{OH})$ vitamin $\mathrm{D}$ (ng/mL). The comparison between genotype groups was designed by the Kruskal-Wallis test and Bonferroni correction to perform multiple comparisons.

studies have reported an association of the rs10766197 polymorphism with the highest mean levels of $25(\mathrm{OH})$ vitamin $\mathrm{D}$ in the healthy Chinese population [48].

We did not find an association between the rs10877012 polymorphism of the CYP27B1 gene and $25(\mathrm{OH})$ vitamin $\mathrm{D}$ levels. However, this association has been reported in other diseases. Ramos-Lopez et al. reported that the rs10877012 C allele was associated with lower levels of $25(\mathrm{OH})$ vitamin $\mathrm{D}$ in a study of patients with gestational diabetes [20].

There could be several reasons why we were not able to find an association between the SNPs and $25(\mathrm{OH})$ vitamin $\mathrm{D}$ levels. These inconsistent results could be derived from differences in population selection and ethnicity. The serum $25(\mathrm{OH})$ vitamin $\mathrm{D}$ levels of the population we studied were much lower than those in other published reports [49-51]. ENSANUT in 2006 reported that approximately $30 \%$ of adults aged 20 to 60 years have hypovitaminosis D [51].

It has been reported in Mexico that $37 \%$ of women of reproductive age have deficient levels and 50\% have insufficient levels of vitamin $\mathrm{D}$ [52].

To date, to the best of our knowledge, this is the first study to analyse the association between these two polymorphisms, 25(OH) vitamin D levels and MS, in Mexican patients, which enabled us to examine the interactions between the CYP2R1 gene, CYPB27 gene, and vitamin D levels to assess how these synergisms may influence MS risk.

There are limitations to the present investigation. First, in relation to the findings of the main effects of individual SNPs and MS risk, these genes were not submitted to an exhaustive examination. However, we cannot exclude the possibility that other gene regions may be important. Second, we did not measure 25-hydroxylase activity. Third, our study was limited to a small sample size.

MS is a multifactorial disease, and this finding supports the notion that risk factors may be relevant only in a proportion of the population with underlying genetic susceptibility [53]. Future investigations that could replicate the findings in this work are necessary to verify the biological precept of the plausibility of gene-environment interactions as they relate to vitamin D and MS risk.

\section{Conclusion}

Reduced serum 25(OH) vitamin D levels were observed in MS patients, although these levels were not associated with disease progression. Carriers of GA + AA rs10766197 of 
the CYP2R1 gene had an increased risk of MS. None of these polymorphisms was associated with severe progression on MS disease.

\section{Data Availability}

The database used to support the findings of this study is available on request. If this database is required, please direct the correspondence to Dr. Ana M. Saldaña-Cruz (ana .saldanac@academicos.udg.mx).

\section{Conflicts of Interest}

The authors declare that there is no conflict of interest regarding the publication of this paper.

\section{Authors' Contributions}

Martinez-Hernandez A contributed to the investigation, writing, original draft, review and editing, and final approval. Perez-Guerrero EE contributed to the methodology, investigation, resources, writing, original draft, review and editing, formal analysis, and final approval. MaciasIslas MA contributed to the methodology, investigation, resources, writing, original draft, review and editing, and final approval. Nava-Valdivia CA contributed to the methodology, investigation, formal analysis, writing, original draft, review and editing, and final approval. VillagomezVega A contributed to the investigation, original draft, review and editing, and final approval. Contreras-Haro B contributed to the writing original draft, review and editing, and final approval. Garcia-Ortega YE contributed to the investigation, writing, original draft, review and editing, and final approval. Esparza-Guerrero Y contributed to the investigation, writing, original draft, review and editing, and final approval. Gallardo-Moya SG contributed to the investigation, original draft, review and editing, and final approval. Gamez-Nava JI contributed to the investigation, original draft, and final approval. Gonzalez-Lopez contributed to the original draft, and final approval. Oliva-Flores E contributed to the investigation, original draft, and final approval. Rodriguez-Jimenez NA contributed to the investigation, original draft, and final approval. Cortes-Enriquez $\mathrm{F}$ contributed to the investigation, original draft, and final approval. Saldaña-Cruz AM contributed to the conceptualization, methodology, investigation, resources, funding acquisition, supervision, project administration, original draft, writing, review and editing, and final approval.

\section{Acknowledgments}

Martinez-Hernandez A, Esparza-Guerrero Y and GallardoMoya SG received scholarships from Consejo Nacional de Ciencia y Tecnologia (Mexico). This project was supported by a grant proportionate for the University of Guadalajara: Fondo para Proyectos de Impulso a la Investigación (PIN 2020).

\section{References}

[1] A. Compston and A. Coles, "Multiple sclerosis," The Lancet, vol. 372, no. 9648, pp. 1502-1517, 2008.

[2] A. J. Thompson, S. E. Baranzini, J. Geurts, B. Hemmer, and O. Ciccarelli, "Multiple sclerosis," The Lancet, vol. 391, no. 10130, pp. 1622-1636, 2018.

[3] Y. Rito, J. Flores, A. Fernández-Aguilar et al., "Vitamin D and disability in relapsing-remitting multiple sclerosis in patients with a Mexican background," Acta Neurologica Belgica, vol. 118, no. 1, pp. 47-52, 2018.

[4] F. D. Lublin, S. C. Reingold, J. A. Cohen et al., "Defining the clinical course of multiple sclerosis: the 2013 revisions," Neurology, vol. 83, no. 3, pp. 278-286, 2014.

[5] D. Karussis, "The diagnosis of multiple sclerosis and the various related demyelinating syndromes: a critical review," Journal of Autoimmunity, vol. 48-49, pp. 134-142, 2014.

[6] M. Macías Islas and E. Ciampi, "Assessment and impact of cognitive impairment in multiple sclerosis: an overview," Biomedicines, vol. 7, no. 1, p. 22, 2019.

[7] N. Ghasemi, S. Razavi, and E. Nikzad, "Multiple sclerosis: pathogenesis, symptoms, diagnoses and cell-based therapy citation: Ghasemi N, Razavi Sh, Nikzad E. Multiple sclerosis: pathogenesis, symptoms, diagnoses, and cell-based therapy," Cell Journal (Yakhteh), vol. 19, no. 1, pp. 1-10, 2017.

[8] A. Ascherio, K. L. Munger, R. White et al., "Vitamin D as an early predictor of multiple sclerosis activity and progression," JAMA Neurology, vol. 71, no. 3, pp. 306-314, 2014.

[9] T. J. Littlejohns, W. E. Henley, I. A. Lang et al., "Vitamin D and the risk of dementia and Alzheimer disease," Neurology, vol. 83, no. 10, pp. 920-928, 2014.

[10] K. L. Munger, J. Åivo, K. Hongell, M. Soilu-Hänninen, H. M. Surcel, and A. Ascherio, "Vitamin D status during pregnancy and risk of multiple sclerosis in offspring of women in the Finnish Maternity Cohort," JAMA Neurology, vol. 73, no. 5, pp. 515-519, 2016.

[11] E. A. Dziedzic, J. S. Gasior, M. Pawłowski, and M. Dabrowski, "Association of vitamin D deficiency and degree of coronary artery disease in cardiac patients with type 2 diabetes," Journal of Diabetes Research, vol. 2017, Article ID 3929075, 11 pages, 2017.

[12] J. M. Lemire, J. S. Adams, R. Sakai, and S. C. Jordan, "1 alpha,25-Dihydroxyvitamin D3 suppresses proliferation and immunoglobulin production by normal human peripheral blood mononuclear cells," The Journal of clinical investigation, vol. 74, no. 2, pp. 657-661, 1984.

[13] S. Chen, G. P. Sims, X. X. Chen, Y. Y. Gu, S. Chen, and P. E. Lipsky, "Modulatory effects of 1,25-dihydroxyvitamin D 3 on human B cell differentiation," The Journal of Immunology, vol. 179, no. 3, pp. 1634-1647, 2007.

[14] C. Scazzone, L. Agnello, G. Bivona, B. Lo Sasso, and M. Ciaccio, "Vitamin D and genetic susceptibility to multiple sclerosis," Biochemical Genetics, vol. 59, no. 1, pp. 1-30, 2021.

[15] J. B. Cheng, M. A. Levine, N. H. Bell, D. J. Mangelsdorf, and D. W. Russell, "Genetic evidence that the human CYP2R1 enzyme is a key vitamin D 25-hydroxylase," Proceedings of the National Academy of Sciences, vol. 101, no. 20, pp. 77117715, 2004.

[16] E. Axen, H. Postlind, H. Sjoberg, and K. Wikvall, "Liver mitochondrial cytochrome P450 CYP27 and recombinantexpressed human CYP27 catalyze 1 alpha-hydroxylation of 
25-hydroxyvitamin D3," Biochemistry, vol. 91, no. 21, pp. 10014-10018, 1994.

[17] T. Shinki, H. Shimada, S. Wakino et al., "Cloning and expression of rat 25-hydroxyvitamin D3-1 -hydroxylase cDNA," Biochemistry, vol. 94, no. 24, pp. 12920-12925, 1997.

[18] A. R. Baker, D. P. Mcdonnellt, M. Hughest, T. M. Crisp, D. J. Mangelsdorft, M. R. Haussler et al., Biochemistry §Present address, vol. 85, Garvan Institute of Medical Research, Saint Vincents Hospital, New South, 1988.

[19] L. Agnello, C. Scazzone, B. Lo Sasso et al., "VDBP, CYP27B1, and 25-hydroxyvitamin D gene polymorphism analyses in a group of Sicilian multiple sclerosis patients," Biochemical Genetics, vol. 55, no. 2, pp. 183-192, 2017.

[20] E. Ramos-Lopez, H. Kahles, S. Weber et al., "Gestational diabetes mellitus and vitamin D deficiency: genetic contribution of CYP27B1 and CYP2R1 polymorphisms," Diabetes, Obesity and Metabolism, vol. 10, no. 8, pp. 683-685, 2008.

[21] S. C. Sánchez, "Mestizaje y características físicas de la población mexicana," Arqueología Mexicana, vol. 65, pp. 64-67, 1996.

[22] J. F. Kurtzke, "On the origin of EDSS," Multiple Sclerosis and Related Disorders, vol. 4, no. 2, pp. 95-103, 2015.

[23] R. Bakshi, A. J. Thompson, M. A. Rocca et al., "MRI in multiple sclerosis: current status and future prospects," The Lancet Neurology, vol. 7, no. 7, pp. 615-625, 2008.

[24] G. Arrambide, M. Tintore, C. Espejo et al., "The value of oligoclonal bands in the multiple sclerosis diagnostic criteria," Brain, vol. 141, no. 4, pp. 1075-1084, 2018.

[25] W. S. Cendrowski, "Progression index and disability status in multiple sclerosis: a resurvey of 207 patients in central Poland," Schweizer Archiv für Neurologie und Psychiatrie, vol. 137, no. 4, pp. 5-13, 1986.

[26] S. MWer, D. Dykes, and H. Polesky, "A Simple Salting out Procedure for Extracting DNA from Human Nucleated Cells," Nucleic Acids Research, vol. 15, 1987.

[27] K. J. Livak, "Allelic discrimination using fluorogenic probes and the $5^{\prime}$ nuclease assay," Genetic Analysis, vol. 14, no. 5-6, pp. 143-149, 1999.

[28] R. Core, Team. A Language and Environment for Statistical Computing, RC Team, 2021.

[29] M. Stevenson, T. Nunes, C. Heuer et al., epiR: Tools for the Analysis of Epidemiological Data, R package version 2.0.38, 2021.

[30] H. Wickham, ggplot2: Elegant Graphics for Data Analysis, Springer-Verlag, New York, 2016.

[31] R. Shinkyo, T. Sakaki, M. Kamakura, M. Ohta, and K. Inouye, "Metabolism of vitamin D by human microsomal CYP2R1," Biochemical and Biophysical Research Communications, vol. 324, no. 1, pp. 451-457, 2004.

[32] M. Wjst, J. Altmüller, T. Faus-Kessler, C. Braig, M. Bahnweg, and E. André, "Asthma families show transmission disequilibrium of gene variants in the vitamin D metabolism and signalling pathway," Respiratory Research, vol. 7, no. 1, p. 7, 2006.

[33] J. H. Laursen, H. B. Søndergaard, A. Albrechtsen et al., "Genetic and environmental determinants of 25hydroxyvitamin D levels in multiple sclerosis," Multiple Sclerosis Journal, vol. 21, no. 11, pp. 1414-1422, 2015.

[34] S. R. Oliveira, A. N. C. Simão, D. F. Alfieri et al., "Vitamin D deficiency is associated with disability and disease progression in multiple sclerosis patients independently of oxidative and nitrosative stress," Journal of the Neurological Sciences, vol. 381, no. 381, pp. 213-219, 2017.
[35] K. L. Munger, L. I. Levin, B. W. Hollis, N. S. Howard, and A. Ascherio, "Serum 25-hydroxyvitamin D levels and risk of multiple sclerosis," Jama, vol. 296, no. 23, p. 2832, 2006.

[36] J. M. Fletcher, S. J. Lalor, C. M. Sweeney, N. Tubridy, and K. H. Mills, "T cells in multiple sclerosis and experimental autoimmune encephalomyelitis," Clinical and Experimental Immunology, vol. 162, no. 1, pp. 1-11, 2010.

[37] L. Legroux and N. Arbour, "Multiple sclerosis and T lymphocytes: an entangled story," Journal of Neuroimmune Pharmacology, vol. 10, no. 4, pp. 528-546, 2015.

[38] M. Goudarzvand, M. Javan, J. Mirnajafi-Zadeh, S. Mozafari, and T. Tiraihi, "Vitamins E and D3 attenuate demyelination and potentiate remyelination processes of hippocampal formation of rats following local injection of ethidium bromide," Cellular and molecular neurobiology, vol. 30, no. 2, pp. 289299, 2010.

[39] F. Chiuso-Minicucci, L. L. W. Ishikawa, L. A. N. Mimura et al., "Treatment with vitamin D/MOG association suppresses experimental autoimmune encephalomyelitis," PLoS One, vol. 10, no. 5, article e0125836, 2015.

[40] L. A. N. Mimura, F. Chiuso-Minicucci, T. F. C. Fraga-Silva et al., "Association of myelin peptide with vitamin D prevents autoimmune encephalomyelitis development," Neuroscience, vol. 317, no. 317, pp. 130-140, 2016.

[41] J. Matías-Guíu, C. Oreja-Guevara, J. A. Matias-Guiu, and U. Gomez-Pinedo, "Vitamina D y remielinizacion en la esclerosis multiple," Neurología (English edition), vol. 33, no. 3, pp. 177-186, 2018.

[42] S. V. Ramagopalan, D. A. Dyment, M. Z. Cader et al., "Rare variants in the CYP27B1 gene are associated with multiple sclerosis," Annals of Neurology, vol. 70, no. 6, pp. 881-886, 2011.

[43] S. Yu, Y. Feng, C. Qu et al., “_CYP27B1_as an instrument gene to investigate the causal relationship between vitamin $\mathrm{D}$ deficiency and obesity: a family-based study," European Journal of Clinical Nutrition, vol. 74, no. 5, pp. 806-810, 2020.

[44] K. C. Simon, K. L. Munger, Xing Yang, and A. Ascherio, "Polymorphisms in vitamin D metabolism related genes and risk of multiple sclerosis," Multiple Sclerosis, vol. 16, no. 2, pp. 133138, 2010.

[45] J. Ahn, K. Yu, R. Stolzenberg-Solomon et al., "Genome-wide association study of circulating vitamin D levels," Human Molecular Genetics, vol. 19, no. 13, pp. 2739-2745, 2010.

[46] T. J. Wang, F. Zhang, J. B. Richards et al., "Common genetic determinants of vitamin D insufficiency: a genome-wide association study," The Lancet, vol. 376, no. 9736, pp. 180-188, 2010.

[47] A. Arabi, N. Khoueiry-Zgheib, Z. Awada et al., "CYP2R1 polymorphisms are important modulators of circulating 25-hydroxyvitamin D levels in elderly females with vitamin insufficiency, but not of the response to vitamin D supplementation," Osteoporosis International, vol. 28, no. 1, pp. 279-290, 2017.

[48] Z. Zhang, J. W. He, W. Z. Fu, C. Q. Zhang, and Z. L. Zhang, "An analysis of the association between the vitamin $D$ pathway and serum 25-hydroxyvitamin D levels in a healthy Chinese population," Journal of Bone and Mineral Research, vol. 28, no. 8, pp. 1784-1792, 2013.

[49] R. Jorde, M. Witham, W. Janssens et al., "Vitamin D supplementation did not prevent influenza-like illness as diagnosed retrospectively by questionnaires in subjects participating in randomized clinical trials," Scandinavian Journal of Infectious Diseases, vol. 44, no. 2, pp. 126-132, 2012. 
[50] S. I. Hassanein, M. A. Abu El Maaty, H. M. Sleem, and M. Z. Gad, "Triangular relationship between single nucleotide polymorphisms in the CYP2R1 gene (rs10741657 and rs12794714), 25-hydroxyvitamin d levels, and coronary artery disease incidence," Biomarkers, vol. 19, no. 6, pp. 488-492, 2014.

[51] M. Flores, L. M. Sánchez-Romero, N. Macías, A. Lozada, E. Díaz, and S. Barquera, Concentraciones séricas de vitamina $D$ en niños, adolescentes y adultos mexicanos, Resultados de la ENSANUT, 2006.

[52] A. Contreras-Manzano, S. Villalpando, and R. Robledo-Pérez, "Vitamin D status by sociodemographic factors and body mass index in Mexican women at reproductive age," Salud Pública de México, vol. 59, no. 5, pp. 518-525, 2017.

[53] A. I. Ruiz-Ballesteros, M. R. Meza-Meza, B. Vizmanos-Lamotte, I. Parra-Rojas, and U. de la Cruz-Mosso, "Association of vitamin D metabolism gene polymorphisms with autoimmunity: evidence in population genetic studies," International Journal of Molecular Sciences, vol. 21, no. 24, pp. 9626-9724, 2020. 